\title{
The Geo CPS Platform for Designing Smart Cities
}

\author{
Wanglin YAN ${ }^{\mathrm{a}, 1}$, Yasushi KIYOKI ${ }^{\mathrm{a}}$ and Yoshifumi Murakami ${ }^{\mathrm{b}}$ \\ ${ }^{a}$ Graduate School of Media and Governance, Keio University \\ ${ }^{b}$ Ad-sol Nissin Corporation
}

\begin{abstract}
The authors have developed the Geo CPS platform, which incorporates the advantages of cyber-physical systems, geographic information systems, and tangible user interfaces, and provides a platform to connect the three components for interactive sensing, processing, and actuation in smart city development. It can be also applied for education in environmental and disaster management, as a tool for technical training, or as a testbed for business solutions.
\end{abstract}

Keywords. IoT, Tangible GIS, smart city, operating structure

\section{Introduction}

The widespread use of the Internet and mobile devices enables the Internet of Things (IoT) and the Internet of Everything (IoE) to capture the appearance and movement of people and objects anytime and anywhere [1]. From this information, it is possible to analyze movements and patterns of people and objects and predict what will happen next. This information can then be fed back into the physical space to support human decisionmaking in real time. For example, drones can automatically take off and land anywhere, anytime, and collect and process images in real time. Self-driving cars are expected to appear on public roads in the near future. These examples foreshadow the arrival of a new type of information system, referred to as a cyber-physical system (CPS), where the cyber and physical spaces work together seamlessly in real time [2, 3, 4].

The CPS concept is being actively discussed in areas as diverse as civil engineering and construction, urban management, and transportation [5]. However, compared to its original use in factories or other closed environments, the use of CPS in open environments tends to be more complex, because most of the work is done in outdoor conditions. For this reason, the development of CPS solutions needs to involve intensive communications from the initial stage, which calls for innovative platforms and user interfaces.

The most common interfaces for geographic information are analog maps and graphic user interfaces (GUI) on electronic monitors. Spherical projection devices such as the digital globe at Japan's National Museum of Emerging Science and Innovation (MIRAIKAN) have also been developed, but they have not gone much beyond the realm of flat electronic display devices. Recently, GIS content is being projected on topographical models, a valuable way to stimulate discussion around a model in a

${ }^{1}$ Corresponding Author, Wanglin Yan, Graduate School of Media and Governance, Keio University, 5322 Endo, Fujisawa City, Kanagawa 252-0816, Japan; E-mail: yan@sfc.keio.ac.jp. 
realistic manner. However, without the ability to respond simultaneously to discussions, that approach is not as interactive and easy to operate as it could be. To address those limitations, tangible user interfaces (touchable user interfaces, or TUIs for short) have been developed that combine $3 \mathrm{D}$ models and map projections $[6,7]$. TUIs are $2 \mathrm{D}, 2.5 \mathrm{D}$, or 3D mockups that utilize augmented reality to provide an interactive space where multiple users can discuss their ideas intuitively in an environment that blends the cyber and physical worlds $[8,9,10]$.

While CPS aims to monitor information and control devices in physical space, TUI attempts to promote multiple-user communication with a mock-up of the physical space. Although they work at different scales, CPS and TUI share the same basic idea of connecting the cyber and physical spaces. With this in mind, the authors have developed the Geo CPS platform [11], which incorporates the advantages of CPS, GIS, and extended TUI (XTUI) [12], and integrates data sensing and actuation of CPS in the physical space, processing of GIS data in the cyber space, and communication with XTUI in the social space (a meeting room). Essentially, the Geo CPS platform is an integration of the three spaces to form a systematic environment for participatory smart city system design and communications. It can be applied for education in environmental and disaster management, as a tool for technical training using GIS and CPS, or as a testbed for business solutions.

Based on the concepts proposed in previous publications [11, 12], this article clarifies the architecture of the Geo CPS platform and describes the functions of the operating structure which we refer to as iSPA. The latest experiment in an Urban Living Lab for a smart city project is presented in the last section.

\section{Concept of the Geo CPS Platform}

In the geographic dimension, information is obtained in physical space by various tools at different heights, from satellite to ground level. The data is managed in cyber space by databases, analyzed by GIS, or modelled by CAD, and then passed on to graphic devices or printers as illustrated in Figure 1. With the advent of IoT devices and everincreasing speed of computer processing, the cycle of observation, storage, processing, and reproduction is becoming faster and finer. The deep learning and structuring of this accumulated data allow us to acquire more sophisticated knowledge and to respond interactively between the cyber and physical spaces.

The technological elements in the Geo CPS platform are not entirely new. Systems to obtain real-time location information from users in the physical space and overlay it with geographic information in the cyber space have been around for a long time. Car navigation systems are the best example of this. Vehicle location, traffic conditions, and the surrounding environment in the physical space are acquired and monitored online, processed and analyzed in real time in the cyber world, and used to direct the actions of people and moving objects. Within Geo CPS, Yan and Sakairi (2019) made a distinction between pseudo-CPS and true-CPS, with the former imposing layers of geospatial datasets on-screen only, and the latter combining those layers into one digital model [11]. The Geo CPS platform integrates the physical space and cyber space. To do this well, the interface between cyber and physical spaces is critical. To cover this requirement, an eXtended TUI (referred to as XTUI) was introduced [12]. 


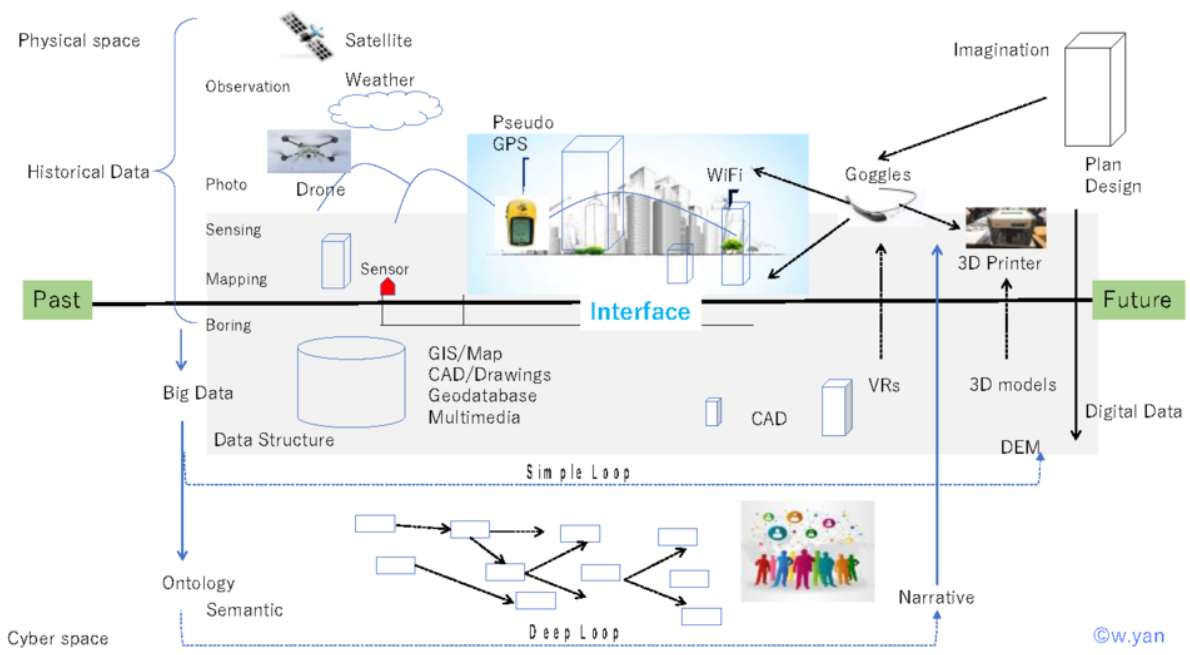

Figure 1. The physical world and cyber world in geographic dimensions (Revised by authors from previous version [11])

\section{System Architecture and Functions}

\subsection{System Architecture}

To utilize the respective advantages of the three spaces, the Geo CPS platform integrates the physical space with sensors, the cyber space in geodatabase, and the social space of XTUI for communications in system development. Figure. 2 shows the system architecture. By analyzing the system components, we discover that these three spaces can each be conceptualized in four layers: platform, network, applications, and users. The physical space is built upon physical terrain, infrastructure, private and public services, and users. The cyber space consists of computer hardware and software, the Internet, intelligent algorithms, and operators. The XTUI is composed of tangible tables, network connections, intuitive interfaces, and multiple participants.

The uniqueness of this structure is that the three spaces are connected to each other by key layers, namely, the network infrastructure and the Internet as the layer between physical and cyber spaces; topology, between physical and social spaces; and digital content and algorithms, between cyber and social spaces. These key layers express the advantages of the three spaces and their specific roles in the platform. Meanwhile, communications between the spaces are realized by iSPA, an extension of the concept of SPA [13], where, "S" stands for sensing in physical space, "P" for processing in the cyber space, "A" for actuation by cyber space or actions inspired by communications in social space, and "i" for intuitive and interactive communication within the cyberphysical-social system. For example, iSPA gathers multi-dimensional information from the local environment and delivers it to the Internet in real time. The information is organized in a layer-based geo-database for cross-scale and cross dimensional analysis and simulation. The contents and algorithms are presented via the XTUI system.

With the Geo CPS platform, communications in a traditional CPS and sensing network can be mocked up in a tangible landscape and visualized in a $3 \mathrm{D}$ model. 
Modification of the mockup can be detected automatically with intelligent algorithms in cyber space. In this way, for town planners, system developers and participants of the project, the design of smart city systems could be simulated in advance of installation similar with the physical mock-up of an urban and architectural development project before construction.

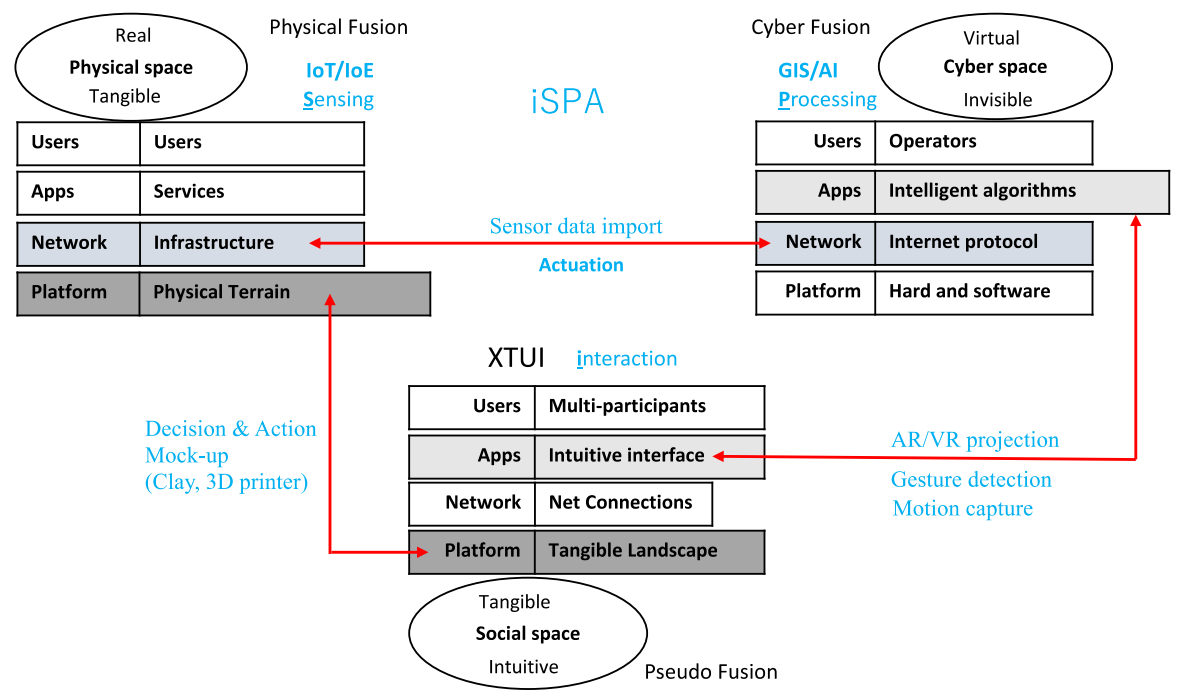

Figure 2. Geo CPS platform's system architecture (Revised by author from previous version [12])

\subsection{System Functions}

Figure 3 shows the functions of the Geo CPS operating structure. For this depiction of iSPA (left), the sensing and actuation layers are placed at the bottom while processing and interaction are placed at the top. The gray boxes represent classes of functions in which sensors and devices are grouped by network and communication protocols. Functions related to cyber space (e.g., data management, data mining, machine learning) are in close proximity to GIS, map projection and AR/VR (augmented reality, virtual reality) $[14,15]$; XTUI brings user services visually to the platform on top of $2.5 \mathrm{D}$ and 3D mockups of the physical space. The tangible landscape is enhanced by GIS and AR/VR simulations. Tangible objects could be mockups of buildings and facilities, etc. Multiple participants can touch the 2.5D and 3D objects to explore the depth of the cyber space using interfaces such as REST and SPARQL and customized application programming interfaces (APIs).

User services could be as diverse as transportation planning, energy and environmental management, innovation, social revitalization, and human health and security. Issues in each of these fields can be expressed in terms of applications in the physical space by integrating information from the inside and interaction with the outside. Work scenarios such as water flow, object detection, thermal simulation, and urban access assessment provide some basic tools for solution development. On the other hand, the Geo CPS platform with XTUI can be used to study ideas, feasibility, and applicability. 


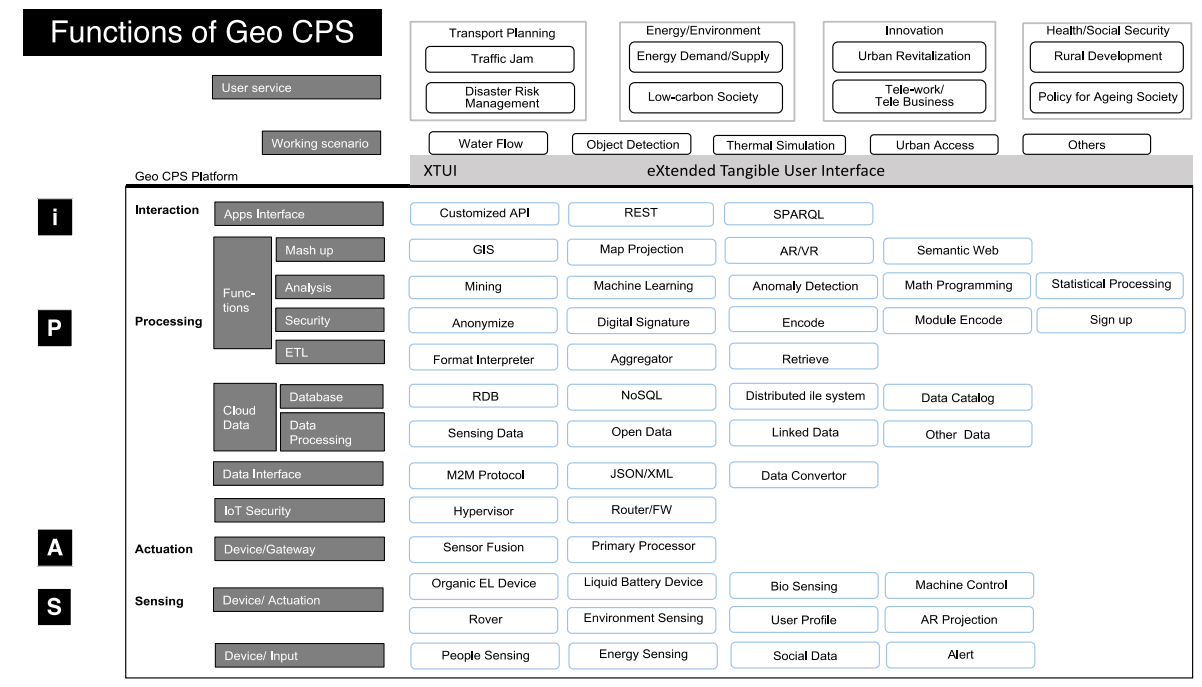

Figure 3. System functions in the Geo CPS platform

(Revised by author from previous version [12])

\section{Prototype and Social Experiment}

In urban planning and design, map projection is often used as a tool to support public participation. In those cases, TUI is used as a tool for visualizing cyber content. For example, Maquil et al. reported on the design and implementation of Geospatial Tangible User Interfaces (GTUIs) to support stakeholder participation in collaborative urban planning $[15,18]$.

The authors are experimenting with the Geo CPS platform by bringing it into our Urban Living Lab. An Urban Living Lab (ULL) is a geographic or institutional place or approach where researchers, citizens, businesses, and governments come together to collaborate, and it is often set up and used for various types of planning and designing, as well as for testing corporate products and solutions [16, 17]. Recently, the concept of the Urban Living Lab has been proposed for use in participatory system design.

The City of Yokohama has developed as a port city, and its waterfront area has played a major role as a new commercial center in the metropolitan region. In the western part of the city, large residential areas have been built as bedroom communities for Tokyo. Many of these residential areas were planned and constructed during the postwar period of rapid economic growth, but the population is now declining, and infrastructure is aging, so the time has come to upgrade infrastructure. To examine this situation, the City of Yokohama partnered with Tokyu Corporation (a major private railway company) to open the WISE Living Lab (WLL) in 2017 on a property owned by the company near Tama Plaza Station in an urban planning effort for the next-generation suburban town (NST). WLL is a place where residents, government, companies, and universities can communicate about local issues, think together about solutions, and co-create the outcomes. The WISE acronym encapsulates the vision of Well-being, Intelligent, Smart and Ecology. We participated in this project and launched a collaborative initiative with 
stakeholders to redesign the living environment in a suburban city. A Geo CPS prototype was set up at WLL to improve users' understanding of the local environment (Figure 4).

The physical space in this case is the outdoor community itself, the cyber space is the cloud GIS server, and the social space is the WLL supported by the XTUI. The XTUI consists of a personal computer, a projector, an infrared camera to detect gestures, and a designed frame. Temperature and air quality sensors are installed in the physical indoor and outdoor areas of the living lab. Three types of sensors are installed: the commercially available Air Egg, one we built based on a Raspberry PI single-board computer, and the coin-sized Leafony developed by the Trillion Node Study Group. The sensing data is sent to cloud data storage through LoRa via an IoT gateway. The data is processed in GIS for mapping and visualization. The processed data will be presented in a cyber space in charts and maps on the project website. Meanwhile, the maps are projected onto a 3D model of the region through the Internet. This allows participants to know the status of the environment on the spot, and the results can be reflected in the XTUI in real time. Participants can manipulate the ground surface made of clay or sand, 3D printed blocks, buildings, and road pavement. The participant's manipulation is captured by an infrared camera and thermal effect of the modification is projected.

In the cyber system, the walkability of the area is calculated and displayed on a monitor. It is then projected onto a 3D model and displayed with red indicating high accessibility along the road network. The user can relocate facilities by moving tangible icons of the facilities on the table. This interaction is done in real time, so the results of changes can be viewed immediately. Overall, this XTUI is beneficial for users to better understand their living environment and built environment and consider ways to improve them.

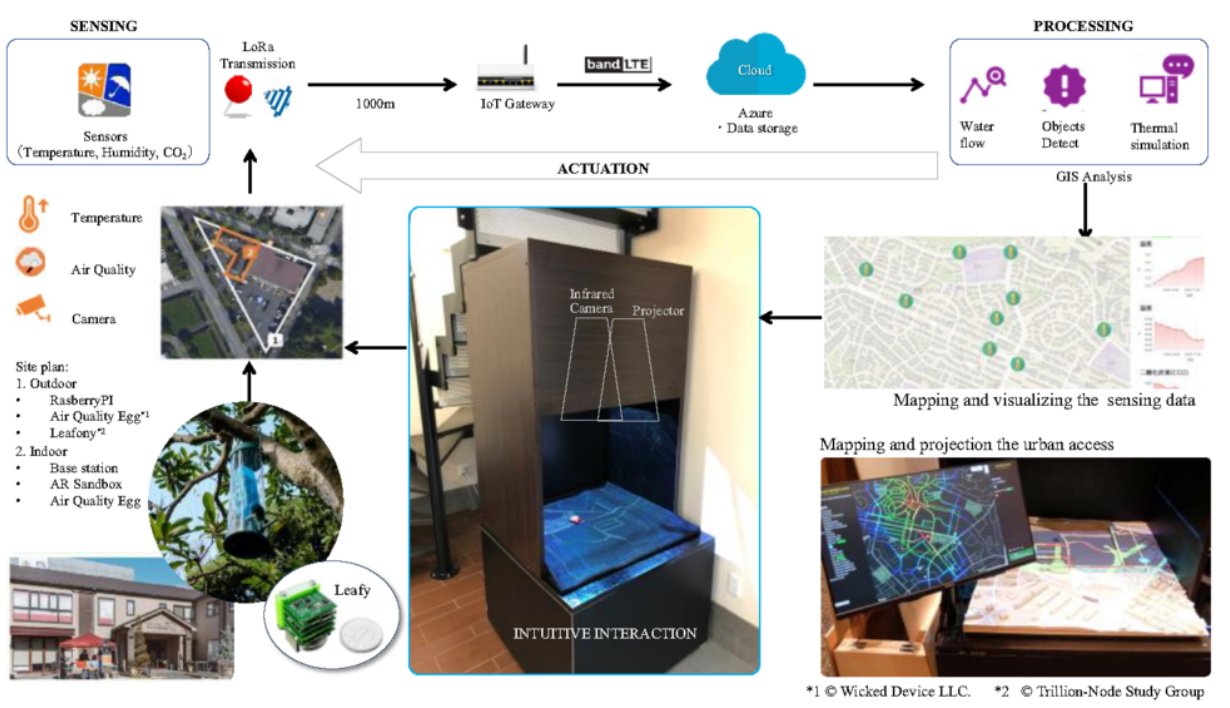

Figure 4. Prototype and experiment

(Revised by author from previous version [12]) 


\section{Conclusions}

This article focused on the integration of geo-technology, IoT and TUI, and presents a Geo CPS platform with eXtended TUI (XTUI) that enables intuitive interaction in cyber, physical and social spaces.

Nowadays, elemental technologies such as GIS, AR/VR, and GNSS (global navigation satellite systems) have made significant advances and become mainstream in the information society, and emerging technologies such as IoT and CPS are driving a new wave of industrial innovation in what has been referred to as Society 5.0. The Geo CPS platform aims to bridge gaps between advanced technologies, geospatial industries, and practical social issues. The platform, consisting of CPS, GIS, and XTUI, leverages the advantages of elemental geospatial technologies and provides a new perspective, focusing on interactions in physical, cyber, and social spaces. The application of the Geo CPS platform in the Urban Living Lab exemplifies an implementation model with community involvement that visibly displays the physical environment on the XTUI table and intuitively promotes interactive discussions. Interactions in the Urban Living Lab can create opportunities for system developers and community leaders to jointly identify problems, co-design solutions, and provide benefits to society.

The Geo CPS platform presented here is still at the early stages of development. Not all its functions have yet been demonstrated and the sensor network is still evolving with the latest availability of sensors, but the design concept is innovative, and we anticipate that it will be able to address many geospatial challenges in smart cities, including social infrastructure management, urban planning, and urban disaster risk response.

\section{Acknowledgements}

This article is the result of research conducted jointly since 2017 by the SFC Institute of Keio University and Ad-sol Nissin Corporation. The prototype has been demonstrated annually at the SFC Open Research Forum. A community trial was conducted by the WISE Living Lab of Tokyu Co. Ltd., with support from the City of Yokohama. The research is also an experiment supported by the grant of Kakenhi titled "Educational practice of ICT-based citizen science for biodiversity conservation through domestic and international collaboration".

\section{References}

[1] Bosch. (2018). 7 factors for getting the most value from your Geo IoT project. https://www.bosch-si.com/geo-iot/geo-iot/homepage-geo-iot.html. Last accessed 2018/03/03.

[2] Hu, L., Xie, N., Kuang, Z., \& Zhao, K. (2012). Review of Cyber-Physical System Architecture. 2012 IEEE 15 th International Symposium on Object/Component/Service-Oriented Real-Time Distributed Computing Workshops, 25-30. https://doi.org/10.1109/ISORCW.2012.15

[3] Lee EA, Seshia SA. (2015). Introduction to Embedded Systems, A Cyber-Physical Systems approach Second Edition, E. A. Lee and S. A. Seshia, Berkley, USA.

[4] Liu, Y., Peng, Y., Wang, B., Yao, S., Liu, Z., \& Concept, A. (2017). Review on Cyber-physical Systems. IEEE/CAA Journal of Automatica SINICA, 4(1), 27-40.

[5] Yang Lu (2017) Yang Lu (2017) Cyber Physical System (CPS)-Based Industry 4.0: A Survey, Journal of Industrial Integration and Management VOL. 02, NO. 03. https://doi.org/10.1142/S2424862217500142

[6] Hofstra, H., Scholten, H., Zlatanova, S., \& Scotta, A. (2008). Multi-user tangible interfaces for effective decision-making in disaster management. In S. Nayak \& S. Zlatanova (Eds.), Remote Sensing and GIS 
Technologies for Monitoring and Prediction of Disasters (pp. 243-266). Berlin Heidelberg: SpringerVerlag.

[7] Monostori, L., Kádá, B., Bauernhansl, T., Kondoh, S., Kumara, S., Reinhart, G., Kumara, S. (2016). Cyberphysical systems in manufacturing. CIRP Annals, 65(2), 621-641.

[8] Ishii, H., \& Ullmer, B. (1997). Tangible Bits: Towards Seamless Interfaces between People, Bits and Atoms. In Proceedings of CHI '97, March 22-27, 1997, (pp. 234-241). New York, New York, USA.: ACM Press. https://doi.org/10.1166/jnn.2017.14175.

[9] Ishii, H., Ratti, C., Piper, B., Wang, Y., Biderman, A., \& Ben-Joseph, E. (2004). Bringing clay and sand into digital design - continous tangible user interfaces. BT Technology Journal, 22(4), 287-299. https://doi.org/10.1023/B:BTTJ.0000047607.16164.16

[10] Harmon, Brendan A., Petrasova, A., Petras, V., Mitasova, H., \& Meentemeyer, R. (2018). Tangible topographic modeling for landscape architects. International Journal of Architectural Computing, 16(1), 4-21. https://doi.org/10.1177/1478077117749959

[11] Yan, W., \& Sakairi, T. (2019). Geo CPS: Spatial challenges and opportunities for CPS in the geographic dimension. Journal of Urban Management, 8(3), 331-341. https://doi.org/10.1016/j.jum.2019.09.005.

[12] Yan W, Y Murakami, A Yasuda, T Makihara, R Fujimoto, and S Nakayama (2021) Developing the eXtended Tangible User Interface as an Experimental Platform for Geo CPS, in M Sakurai and R Shaw Eds. Emerging Technologies for Disaster Resilience - Practical Cases and Theories, Springer Nature (in press).

[13] Kiyoki Yasushi, Petchporn Chawakitchareon, Sompop Rungsupa, Xing Chen and Kittiya Samlansin (2020) A Global \& Environmental Coral Analysis System with SPA-based Semantic Computing for Integrating and Visualizing Ocean-Phenomena with "5-Dimensional World-Map". In B. Thalheim, M. Tropmann-Frick, H. Jaakkola, Y. Kiyoki (eds). Proceedings of the 30th International Con- ference on Information Modelling and Knowledge Bases (EJC 2020), June 8-9, 2020, Hamburg, Germany.

[14] Maquil, V., De Sousa, L., Leopold, U., \& Tobias, E. (2015). A geospatial tangible user interface to support stakeholder participation in urban planning. In GISTAM 2015 - 1st International Conference on Geographical Information Systems Theory, Applications and Management, Proceedings (pp. 113-120). SCITEPRESS.

[15] Maquil, V., Leopold, U., De Sousa, L. M., Schwartz, L., \& Tobias, E. (2018). Towards a framework for geospatial tangible user interfaces in collaborative urban planning. Journal of Geographical Systems, 20(2), 185-206. https://doi.org/10.1007/s10109-018-0265-6

[16] McCormick, K. and Hartmann, C. (2017) The emerging landscape of urban living labs: Characteristics, practices and examples. https://doi.org/10.1371/journal.pone.0099587.

[17] Thinyane, M., Terzoli, A., Thinyane, H., Hansen, S., \& Gumbo, S. (2012). Living Lab Methodology as an Approach to Innovation in ICT4D: The Siyakhula Living Lab Experience. IST-Africa 2012, 1-9. 